\title{
Salter-Harris Type II Humeral Head Fracture with Kirschner Wire Fixation in a Goat
}

\author{
Darby W. Walmsley ${ }^{1} \quad$ Tania Shaw $^{1} \quad$ Simon T. Kudnig ${ }^{1}$ \\ ${ }^{1}$ Animal Referral Hospital, Melbourne, Australia \\ Address for correspondence Darby W. Walmsley, BVSc, Animal \\ Referral Hospital, Essendon Fields, 72 Hargrave Avenue, Essendon \\ VCOT Open 2020;3:e153-e157. \\ Fields, Melbourne 3041, Australia \\ (e-mail: darby.walmsley@gmail.com).
}

\begin{abstract}
Keywords

- small ruminant

- humerus fracture

- osteosynthesis

The aim of this study was to describe a case of a Salter-Harris type II fracture of the humeral head in a goat repaired with Kirshner wire fixation. A 3-year-old, $52 \mathrm{~kg}$, male, non-domesticated goat was referred for evaluation. Approximately 11 days prior, the goat sustained suspected vehicular trauma, and was found on the side of the road with a non-weight bearing lameness of the right thoracic limb. Orthogonal radiographic assessment identified a Salter-Harris type II fracture of the humeral head with proximolateral displacement of the humerus. The fracture was repaired with multiple Kirschner wire fixation. Orthogonal radiographic assessment of the right humerus at 8 weeks postoperatively revealed implant quiescence with radiographic union of the fracture site. Long-term telephone follow-up at 3 and 6 months postoperatively found the patient was no longer restricted in activity, with free access to the field, and was not displaying an overt lameness. The authors describe a novel fracture configuration in the goat successfully repaired with multiple Kirschner wire fixation.
\end{abstract}

\section{Introduction}

Proximal humeral fractures in small ruminants are seldom and infrequently reported in the veterinary literature. ${ }^{1,2}$ of the humeral fractures described in goats, most are mid-body, diaphyseal fractures that are predominantly the result of vehicular trauma ${ }^{2}$ or traumatic dog attacks. ${ }^{3}$ However, specific behavioural patterns of goats, including their curiosity and climbing instincts, may increase their propensity for sustaining traumatic fractures. ${ }^{3}$ Reported methods of repair of diaphyseal humeral fractures in this species include open reduction and internal fixation with bone plates, screws and/ or intramedullary pins. ${ }^{4}$ Given the location, fracture repair of the humerus is not amenable to external coaptation due to the inability to immobilize the glenohumeral joint.

The proximal humerus of the goat has two centres of ossification: the greater tubercle and the articular head. ${ }^{5,6}$ In males, fusion of the greater tubercle and the articular head initiate and completely fuse in the third year of life; however, fusion may continue until year 5 with definitive continuity to the diaphysis by year 9.5,6 Female goats show a faster

received

April 11, 2020

accepted after revision

August 29, 2020 ossification process than males, with fusion often occurring in the first or second year of life. ${ }^{5,6}$ In comparison, closure of the proximal humeral physes in dogs occurs between 7.5 and 12 months. ${ }^{7,8}$ Uniquely, goat humeri characteristically possess large and elongated greater and lesser tubercles that extend above the head of the humerus. ${ }^{9}$ The aforementioned anatomical variations between domestic animals and ruminants are important for surgical planning and appropriate implant selection.

In companion animals, fracture-separation of the confluent proximal humeral epiphyses (Salter-Harris type I and II) is the most common configuration of proximal humeral fractures in skeletally immature dogs. ${ }^{10-12}$ Fractures of the proximal humeral epiphyses are intrinsically stable given the nature of the fracture interface, ${ }^{12}$ although fractures with fragment displacement generally represent a more challenging repair given its inherent instability. ${ }^{12}$ Isolated SalterHarris type I and II fractures of the humeral head are very rarely reported, ${ }^{12}$ with one case reported in a 4 -year-old Dachshund. ${ }^{13}$ Thus, no ideal fixation method for such fractures has been established. ${ }^{14}$ Conventional methods of repair
License terms

Stuttgart · New York

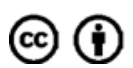


include double transfixing Kirshner wires, or fixation by a lag screw. ${ }^{11}$

To the authors knowledge, Salter-Harris type II fractures of the humeral head and their subsequent repair have not been described in the goat. This report describes the use of triple transfixing Kirshner wires for the treatment of a SalterHarris type II fracture of the humeral head in a goat.

\section{Case Description}

\section{Clinical History, Examination and Diagnostic Imaging}

A 3-year-old, $52 \mathrm{~kg}$, male, non-domesticated goat was referred to a referral veterinary hospital for evaluation. Approximately 11 days prior, the goat sustained suspected vehicular trauma, and was found on the side of the road with a non-weight bearing lameness of the right thoracic limb. The goat was initially transported to an animal rescue facility for supportive care prior to transportation for veterinary assessment.

Findings on physical examination included a temperature of $39.3^{\circ} \mathrm{C}$, a heart rate of $90 \mathrm{bpm}$ and respiratory rate of $60 \mathrm{bpm}$. The goat was non-weight bearing on the right thoracic limb. Swelling of the right proximal humeral region was immediately apparent, with palpation of the proximal humerus eliciting a moderate pain response. Pain and crepitus were identified when the shoulder was manipulated through its range of motion, with normal range of motion moderately restricted. Pain was also appreciated on full extension and full flexion on the right shoulder. Further orthopaedic and neurological examination was unremarkable.

Orthogonal radiography (KVp 80, mAs 6.4, focus film distance: $100 \mathrm{~cm}$ ) of the right thoracic limb was performed, identifying a Salter-Harris type II fracture of the humeral head with caudomedial displacement of the humeral head fragment $18 \mathrm{~mm}$ from normal anatomical positioning (-Fig. 1).

\section{Surgical Technique}

Butorphanol $(0.1 \mathrm{mg} / \mathrm{kg}$ intravenously [IV]) and midazolam $(0.1 \mathrm{mg} / \mathrm{kg} \mathrm{IV})$ were administered as pre-medication. Anaesthesia was induced with a combination of ketamine $(5 \mathrm{mg} / \mathrm{kg}$ IV) and midazolam $(0.1 \mathrm{mg} / \mathrm{kg}$ IV), with anaesthesia maintained with isoflurane. The right thoracic limb was clipped and prepared with chlorhexidine scrub and a $70 \%$ alcohol solution in a sterile manner, and the patient positioned in left lateral recumbency. A craniolateral approach to the right shoulder was performed with incision into the deep brachial fascia, caudal retraction of the acromial head of the deltoid muscle, tenotomy of the infraspinatus muscle and incision into the joint capsule. The joint capsule appeared subjectively thickened with evidence of synovitis. The fracture site was identified and a periosteal elevator was used to advance the humeral head fragment cranially. Due to chronicity of the fracture, accurate reduction and alignment were difficult. Two 7/64 $(2.77 \mathrm{~mm})$ and one $3 / 32(2.38 \mathrm{~mm})$ Kirschner pins were then inserted retrograde from the humeral fracture site proximally to exit the lateral humeral cortex. The pins were then withdrawn until their tips were level with the most proximal aspect of the distal fracture segment. The humeral head was then reduced with a periosteal elevator with concurrent extension

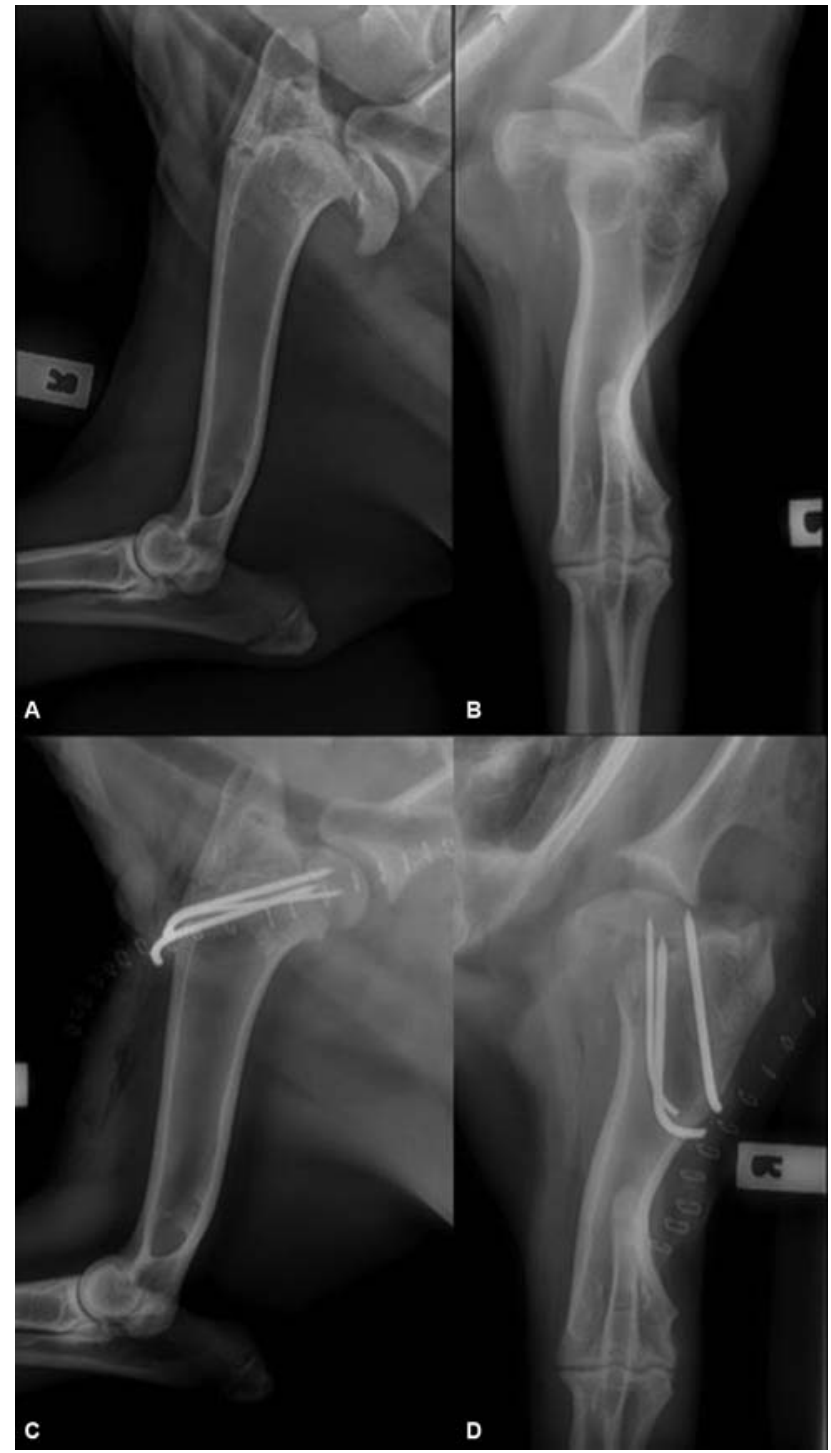

Fig. 1 Preoperative and immediate postoperative radiography (mediolateral projection: (A) and (C); craniocaudal projection: (B) and (D)) of the humerus.

of the shoulder and the pins driven $\sim 11 \mathrm{~mm}$ normograde to capture the humeral head. One pin was observed to exit the cartilage of the humeral head and it was withdrawn until the tip of the pin was below the cartilage surface. The authors did not have access to intraoperative fluoroscopy at the time of surgery. Some capsular attachments were retained in the proximal fracture fragment; however, some attachments were visibly torn. The shoulder was then put through a range of motion and no crepitus or pin impingement could be palpated. The joint was copiously lavaged with sterile saline and the joint capsule closed with $2 / 0$ polydioxanone simple interrupted sutures. The infraspinatus tendon was re-attached with $2 / 0$ polydioxanone horizontal mattress sutures. The brachial fascia was closed with 2/0 PDS simple continuous. The subcutaneous tissue was closed with $3 / 0$ polydioxanone in a simple continuous suture pattern, and the skin closed with 4/ 0 poliglecaprone 25 in a simple continuous intradermal suture pattern. Skin staples were also applied to the cutaneous layer. 
Postoperative radiography (mediolateral and craniocaudal projections) revealed adequate fracture reduction (-Fig. 1); however, medial displacement remained apparent on the cranial-caudal view. The pins were located on the lateral portion of the proximal fracture fragment. One of the pins appeared to be short on postoperative radiography; however, this was the pin that was observed to exit the cartilage of the lateral part of the fragment and was withdrawn just under the cartilage.

\section{Perioperative and Postoperative Management}

Perioperative antibiotic therapy consisted of cefazolin (22 mg/kg IV; Cefazolin-AFT, AFT Pharmaceuticals, North Ryde, NSW, Australia) 30 minutes prior to the first incision, and every 90 minutes during surgery thereafter (total surgery time 1 hour, 52 minutes). Perioperative analgesia included butorphanol $(0.05-0.1 \mathrm{mg} / \mathrm{kg} / \mathrm{h})$ and ketamine $(0.6 \mathrm{mg} / \mathrm{kg} / \mathrm{h})$ constant rate infusions.

Postoperative analgesia consisted of buprenorphine (0.01 mg/kg IV q8h), meloxicam ( $0.1 \mathrm{mg} / \mathrm{kg}$ subcutaneously; Meloxicam, Ilium, Troy Laboratories Australia Pty Ltd, Australia) and a transdermal fentanyl patch $(50 \mu \mathrm{g} / \mathrm{h}$; Durogesic 50, Janssen-Cilag Pty Ltd, Macquarie Park NSW, Australia). Pain scores were performed using the Glasgow composite pain scale every 4 hours with administration of buprenorphine as required until activation of the transdermal fentanyl patch 12 hours following application.

The patient was discharged from hospital the following day, with meloxicam $(0.1 \mathrm{mg} / \mathrm{kg}, 1.5 \mathrm{mg} / \mathrm{mL}$; Meloxicam, Ilium, Troy Laboratories Australia Pty Ltd, Australia) dispensed for oral administration during the postoperative period, as well as penicillin to be injected subcutaneously, both for 7 days postoperatively. At the time of discharge, the patient was non-weight bearing on the operated limb. The application of an adjuvant scapulohumeral stabilization technique, such as a Velpeau-sling, was not elected given specific patient characteristics (non-domesticated farm animal) and difficulty in bandage monitoring. The patient was to be restricted to a small pen for 8 weeks.

\section{Clinical Outcome}

The patient presented at 2 and 8 weeks postoperatively for re-evaluation by their primary care veterinarian. At 2 weeks, the patient presented with a moderate (grade $3 / 5$ ) thoracic limb lameness, with mild pain evident on manipulation of the humerus. At 8 weeks, no lameness was apparent at this time, with range of motion of the shoulder considered within normal limits. No pain or crepitus was appreciated on manipulation of the shoulder. Mediolateral radiographic assessment of the right humerus performed by the primary care veterinarian revealed implant quiescence with radiographic union of the fracture site (-Fig. 2 ).

Telephone and video updates were provided at both 3 and 6 months postoperatively by the client ( - Video 1 ). No visible lameness was apparent via video update, with the owner reporting that the patient was no longer restricted in activity, with free access to the field. Follow-up of the patient was lost thereafter.

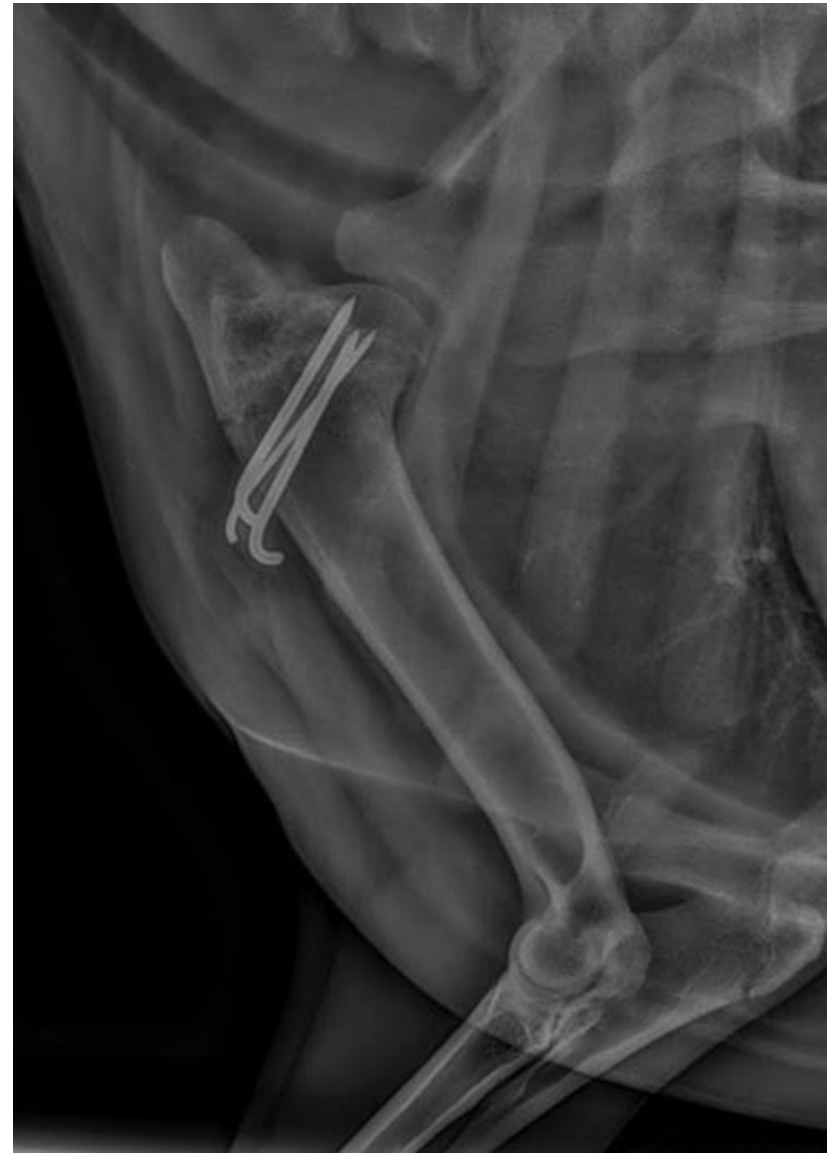

Fig. 2 Radiographs of the humerus obtained 8 weeks postoperatively.

\section{Video 1}

Long-term video follow-up of patient provided by the owner. Online content including video sequences viewable at: https://www.thieme-connect.com/ products/ejournals/html/10.1055/s-0040-1718377.

\section{Discussion}

Proximal humeral epiphyseal fractures are infrequently reported in both the human and veterinary literature. ${ }^{15,16}$ Dissimilarly to that of companion animals, goat humeri characteristically possess unique anatomical variances that result in a dense region of bone located in close proximity to the body, which may further reduce the likelihood of proximal humeral fractures in this species. ${ }^{5-9}$ Epidemiological studies in paediatrics have identified Salter-Harris type II fractures of the proximal humeral epiphysis to be the most common fracture configuration and often the result of high energy trauma. ${ }^{15,16}$ Such trauma typically results in fractures propagating along epiphyseal scar lines, with muscle forces on fracture fragments and limb position at the time of impact also in consideration for fracture type and severity. ${ }^{17}$ Isolated epiphyseal separation 
is rare and occurs typically in newborns and toddlers, ${ }^{15}$ with treatment often conservative and consisting of shoulder immobilization. ${ }^{15}$ Despite previous studies identifying similar outcomes between surgery and non-operative treatment, ${ }^{15,18}$ management of proximal humeral fractures in people has evolved toward favouring surgical intervention, particularly in older patients and those with grossly displaced or angulated fractures. ${ }^{19,20}$ German national guidelines for proximal humeral fractures in people recommend reduction and stabilization by elastic-stable intramedullary nailing in patients over the age of 10 years, with minimally displaced fractures often left untreated due to the highly potent epiphyseal plate. ${ }^{21}$ For patients managed conservatively, proximal humeral remodelling plays an important role in healing and fracture stabilization. Dameron and Reibel ${ }^{20}$ stated that the remodelling capacity of the proximal humerus is age dependent, and thus excellent results are expected in children younger than 11 years of age regardless of degree of displacement and angulation. Conversely, patients older than 11 years with more than 20 degrees of fracture angulation are only expected to achieve partial correction by humeral remodelling. ${ }^{20}$ When treated with primary reduction and Kirschner wire stabilization, multiple studies have demonstrated excellent outcomes without complications in 94.4 to $95 \%$ of cases. ${ }^{19,22}$ When treated conservatively, outcomes are still considered excellent, ranging between 82 and $90.14 \%$ of cases. ${ }^{18,22}$ Similar retrospective studies investigating proximal humeral fractures are currently lacking in veterinary literature, with treatment therefore guided by studies of similar physeal injuries, case reports and clinician experience.

As with many other fracture fixation methods, surgical correction of proximal humeral fractures must adhere to certain principles, including minimizing soft tissue dissection around the fracture fragments, and limiting the number of implants required for stable fixation. ${ }^{23}$ Tenuous retrograde vascular supply to the intracapsular humeral head is often significantly disrupted with proximal humeral fractures, with comminuted and displaced fractures commonly recognized as predisposing risks for anatomical head infarction in people. ${ }^{24}$ Further, any anatomical neck fracture is associated with increased risk of avascular necrosis. ${ }^{24}$ Hertel and colleagues ${ }^{24}$ note that the risk of avascular necrosis in humans increases with medial periosteal disruption and displacement of the proximal segment greater than $2 \mathrm{~mm}$. In the same study, a positive predictive value of $97 \%$ for ischaemia was recognized with a combination of anatomical neck fracture with a displaced medial metaphyseal segment. ${ }^{24}$ In this case, displacement of the humeral head was $18 \mathrm{~mm}$ caudomedially, representing a Neer-Horowitz classification grade III preoperative fracture displacement, ${ }^{15}$ and thus predisposing the patient to significant risk of avascular necrosis of the humeral head. As soft tissue injuries are often more extensive with displaced fractures, the potential for instability or pain despite bone healing, as well as the development of heterotrophic ossification, is increased. ${ }^{24}$

The use of minimally invasive percutaneous pinning techniques has been advocated for use in fractures of the humeral head in an attempt to avoid complications such as implantassociated risks, neurovascular damage and avascular necrosis. $^{23}$ In a study by Boekhout-Ta and colleagues, ${ }^{25}$ closed reduction and fluoroscopic-assisted percutaneous pinning of proximal humeral physeal fractures in three dogs achieved appropriate radiographic healing. Given the degree of humeral head displacement and unique anatomical variances of the goat, percutaneous pinning was not deemed feasible, and therefore open anatomical reduction and fixation were performed. Open anatomic reduction with Kirschner wire fixation was elected in this case based on previously reported long-term results in children that demonstrated good outcomes with no complications in proximal humeral physeal fractures treated with Kirschner wire fixation. This method has also shown to reduce the risks of secondary physeal damage, which may result growth arrest. ${ }^{15}$ The necessity to achieve anatomic fracture reduction was important in this case to avoid instability, progressive osteoarthritis, limb length discrepancies and angular limb deformities. A biomechanical comparison of Kirschner wire fixation on fracture stability in Salter-Harris type I fractures of the proximal humeral physis in a porcine cadaveric model identified that three-pin and two-pin fixation significantly reduced rotational interfragmentary movement compared with one-pin fixation. ${ }^{14}$ Although construct stiffness was not significantly different between any of the pin groups of the study, threepin fixation was elected in this case as it demonstrated increased construct stiffness comparatively to two- and one-pin fixation in the porcine model. ${ }^{14}$

In this report, one pin was observed to inadvertently exit the cartilage of the humeral head and was withdrawn immediately until the tip of the pin was below the cartilage surface. Although this may result in an increased risk of developing progressive osteoarthritis and pain, the authors believe this to likely be insignificant in long term; however, further long-term radiography would be required to substantiate this claim. A limb length discrepancy or angular limb deformity was not apparent on follow-up examination. The patient demonstrated a satisfactory clinical outcome with no overt pain or lameness apparent at 6 months postoperatively. Possible long-term complications beyond our follow-up include implant migration, progressive osteoarthritis, pain and reduced glenohumeral range of motion.

\section{Conclusion}

This report illustrates a successful functional and radiographic outcome of an isolated Salter-Harris type II humeral head fracture with Kirschner wire fixation in a goat. To the authors' knowledge, this is the first report to describe a proximal humeral physeal fracture and its subsequent repair in this species.

\section{Authors' Contributions}

All authors contributed to conception, study design, acquisition of data and data analysis, and interpretation. They also drafted, revised, and approved the submitted manuscript. 


\section{Conflict of Interest \\ None declared.}

\section{References}

1 Kofler J, Hochschwarzer D, Schieder K, Osová A, Vidoni B. [Limb fractures in 32 small ruminants - treatment and outcome]. Tierarztl Prax Ausg G Grosstiere Nutztiere 2017;45(04):201-212

2 Gupta S. Fracture healing using biphasic calcium phosphate with dynamic compression plating in goats. MVSc, \& AH thesis. Jabalpur: Surgery and Radiology. Nanaji Deshmukh Veterinary Science University; 2015

3 Joy B, Venugopal SK. Complications in fracture healing using external skeletal fixation in goats. Int J Sci Res (Ahmedabad) 2014;5(07):1298-1299

4 Matthews J. Diseases of the Goat. 4th edition. Surgical Techniques. Chichester, West Sussex, UK: John Wiley \& Sons Limited; 2016: 364-395

5 Serrano E, Gállego L, Pérez JM. Ossification of the appendicular skeleton in the Spanish Ibex Capra pyrenaica Schinz, 1838 (Artiodactyla: Bovidae), with regard to determination of age. Anat Histol Embryol 2004;33(01):33-37

6 Serrano E, Sarasa M, Perez J, et al. Patterns of epiphyseal fusion in the appendicular skeleton of the Iberian wild goat Capra pyrenaica, and comparisons with other Artiodactyla. Mamm Biol 2010; 76:97-100

7 Sumner-Smith G. Observations on epiphyseal fusion of the canine appendicular skeleton. J Small Anim Pract 1966;7(04):303-311

8 von Pfeil DJ, DeCamp CE. The epiphyseal plate: physiology, anatomy, and trauma. Compend Contin Educ Vet 2009;31(08): E1-E11, quiz E12

9 Makungu M, Merere B. Morphology of the thoracic limb of goat as evidenced by gross osteology and radiology. Anat Histol Embryol 2017;46(06):509-518

10 Longley-Hobbs S. Fractures of the humerus. In: Tobias KM, Johnston SA, eds. Veterinary Surgery: Small Animals. 1st edn. St. Louis, MO: Elsevier Saunders; 2012:820-836

11 Brinker P. Flo. Fractures of the Humerus. Handbook of Small Animal Orthopaedics and Fracture Repair. 5th edition. St. Louis, Missouri: Elsevier Inc; 2016:298-327

12 Perry K, Woods S. Humeral fractures. Companion Anim 2017;22 (08):454-664
13 King SJ. Repair of femoral and humeral fractures of small animals by closed reduction intramedullary pinning. Aust Vet J 1970;46 (05):227-230

14 Ma J, Wang T, Lovric V, Johnson KA, Walsh WR. A biomechanical comparison of Kirschner-wire fixation on fracture stability in Salter-Harris type I fractures of the proximal humeral physis in a porcine cadaveric model. BMC Vet Res 2017;13(01):306. Doi: 10.1186/s12917-017-1225-y

15 Binder H, Schurz M, Aldrian S, Fialka C, Vécsei V. Physeal injuries of the proximal humerus: long-term results in seventy two patients. Int Orthop 2011;35(10):1497-1502

16 Passaretti D, Candela V, Sessa P, Gumina S. Epidemiology of proximal humeral fractures: a detailed survey of 711 patients in a metropolitan area. J Shoulder Elbow Surg 2017;26(12): 2117-2124

17 Majed A, Thangarajah T, Southgate D, et al. The biomechanics of proximal humeral fractures: injury mechanism and cortical morphology. Shoulder Elbow 2018;0(00):1-9

18 Larsen CF, Kiaer T, Lindequist S. Fractures of the proximal humerus in children. Nine-year follow-up of 64 unoperated on cases. Acta Orthop Scand 1990;61(03):255-257

19 Bahrs C, Zipplies S, Ochs BG, et al. Proximal humeral fractures in children and adolescents. J Pediatr Orthop 2009;29(03):238-242

20 Dameron TB Jr, Reibel DB. Fractures involving the proximal humeral epiphyseal plate. J Bone Joint Surg Am 1969;51(02): 289-297

21 Schmittenbecher PP, Blum J, David S, et al. [Treatment of humeral shaft and subcapital fractures in children. Consensus report of the child trauma section of the DGU]. Unfallchirurg 2004;107(01):8-14

22 Hohloch L, Eberbach H, Wagner FC, et al. Age- and severityadjusted treatment of proximal humerus fractures in children and adolescents-a systematical review and meta-analysis. PLoS One 2017;12(08):e0183157

23 Park MC, Murthi AM, Roth NS, Blaine TA, Levine WN, Bigliani LU. Two-part and three-part fractures of the proximal humerus treated with suture fixation. J Orthop Trauma 2003;17(05):319-325

24 Hertel R, Hempfing A, Stiehler M, Leunig M. Predictors of humeral head ischemia after intracapsular fracture of the proximal humerus. J Shoulder Elbow Surg 2004;13(04):427-433

25 Boekhout-Ta CL, Kim SE, Cross AR, Evans R, Pozzi A. Closed reduction and fluoroscopic-assisted percutaneous pinning of 42 physeal fractures in 37 dogs and 4 cats. Vet Surg 2017;46(01):103-110 